\title{
Catastrophic Anachronisms: The Past, Present and Future of Disaster Medicine
}

\author{
K. J. Rinnert, J. G. Wigginton, and P.E. Pepe
}

\section{Introduction}

Disasters, particularly earthquakes, volcanoes, floods, war-related complications, famine and infectious epidemics, have been a part of recorded human experience. From Pompeii to the Johnston Flood and World War II and the Black Plague to the Spanish Influenza, there have been catastrophic occurrences that will not long be forgotten by either legend or history books. Nevertheless, those occurrences were relatively few and far-between before the mid-twentieth century. Indeed, the nature of disasters has changed since then. From terrorists taking advantage of 'new technology' to weather-related events that cause trillions of dollars worth of damages and economic loss, the world has evolved.

In the last 50 to 60 years alone, the risk and frequency of events with multiple injuries and deaths has increased dramatically [1-4] and it is bound to increase logarithmically over the next half century. Not only is the earth more heavily-populated with human beings settling across many more regions of the planet, but there are also larger concentrations of human inhabitants dependent on other resources for sustenance. Dependency on others for complicated food supply chains, refrigeration, fuel and power sources and public health hygiene (sewage handling), makes human populations more vulnerable than ever before and less self-sufficient. In addition, these large, vulnerable population centers are often concentrated in high-risk locales such as metropolitan cities where very frequent and multiple person-to-person contacts occur. Often they are along disaster prone areas, be they in coastline or sub-sea level reclaimed lands.

In addition, the world now faces a broadened spectrum of disasters, ranging from terrorist bombings, unconventional wars, nuclear releases, transportational mishaps, worldwide infectious disease epidemics and chemical discharges (technology-generated) to floods, famine, earthquakes, tornadoes, cyclones, fires, and other 'natural disasters' $[2,4]$. It may also be that human technology has increased the likelihood of more severe and more frequent natural disasters. Whether it is global warming (from industry and omnipresent internal combustion machines) leading to stronger and more frequent hurricanes or the jumbo jet that rapidly translocates deadly influenza viruses around the planet, the threat continues to evolve far beyond the world of our grandparents.

Therefore, although famine, war, plagues, earthquakes and such threats have darkened the human experience, the on-going spiral of human populations, the rapid growth of technology, relatively easy world-wide travel for millions and the exponential expansion of at-risk industries and domiciles have now conspired to dramatically increase human exposure to catastrophic events. In turn, the potential for 
casualties resulting from each incident is more likely to be much larger [2] and the magnitude of the economic costs beyond our current comprehension.

The recent undersea earthquake in the Indian Ocean that sent towering, 700 kilometer per hour tsunamis across Southern Asia resulted in a tremendous number of deaths and morbidity because of the sheer volume of exposures in vulnerable sites. The 1950s seaside fishing villages of 5,000 local inhabitants had become cosmopolitan, internationally-populated, destination resorts hosting hundreds of thousands supported by multi-national industries and technology. Such expanding population bases living and working in vulnerable situations along the at-risk seashores such as Phuket, Thailand, or in major metropolitan settlements built below sea level such as New Orleans, USA, provided unprecedented fodder for disaster, regardless of continent. Likewise, the recent earthquake in Pakistan that killed tens of thousands in a few minutes might not have had such an effect 50 years ago when such concentrated populations centers did not exist.

If that were not enough, beyond a higher risk for casualties and frequency of events, the events have now become global with far-reaching psychological and sociological impact. While still true in some respects, the old adage that "all disasters are local" may have become somewhat anachronistic since the mid- $20^{\text {th }}$ Century. Although the Christmas 2004 tsunami disaster occurred in Southern Asia, it affected many other countries worldwide because those nation's citizens and many international businesses were involved. Mass air travel, economic strategic initiatives and evolving technology have all changed the face of 'local' disasters. With the rapid growth of air travel and relatively affluent worldwide economies, travel and sightseeing have morphed from an occasional pastime of the very privileged and the eclectic adventurer into a prevailing norm of worldwide mass tourism. Likewise, industries have also become global, often seeking heavily-populated sites where labor may be more economical and local workforces readily available to work for lower wages. A large number of the victims of the Indian Ocean tsunamis were foreign nationals operating businesses and many, many tourists, including celebrities, were caught up in this Asian calamity as well. Thus, in many ways, disasters throughout the world have now become multinational in nature, having global impact, an impact amplified by mass media coverage and internet streaming of the events.

Even so, a disaster in New Orleans involving mainly geography of only a few tens of kilometers, had ramifications in major cities hundreds of miles of way. Both before, and especially after, the strike of Hurricane Katrina in late August of 2005, many tens of thousands of evacuees had to settle in Dallas and Houston affecting resources in those cities and taxing the local medical system. For example, one Dallas shelter (750 kilometers from New Orleans) treated twice the number of medical problems and emergencies (among the evacuees) than it would on a day-to-day basis in the city's main trauma center. This sudden surge in medical problems required the establishment of a 'field' medical facility for more than two weeks after the storm had dissolved. Not only did those patients have their day-to-day medical, dental and psychological needs, but they were now at greater risk in terms of compromised immune systems (nutrition/sleep deprived and psychologically-stressed), but also because of crowded conditions.

From another perspective, despite the relatively fewer number of deaths than the 2004 tsunami or the 2005 Pakistani quake, the September 11, 2001 attack on the World Trade Center in New York could be considered a multi-national event because of the hundreds of persons from dozens of countries working there. It also 
pointed out the concepts of modern vulnerability. Not only were the victims highly concentrated in one building making it easier to target and cause many casualties, but modern technology facilitated the incident. A large modern transportation device laden with explosive fuel made such a dramatic disaster possible, one that would not have occurred to such an extent before 1950 .

While such technology makes a local disaster global in nature, the same technology can bring a global disaster to each locality. The threat of worldwide spread of contagious disease, both naturally-occurring and malicious (bioterrorist) promulgation $[5,6]$ is accelerated not only by exponential population growths, but the air ship as well. Again, with more people on the planet who have the potential to become an infected vector and with more global concentrations of highly-mobile populations moving around in jet aircraft (and thus more opportunities for exposure), the risk for pandemics has clearly become greater than ever before. Although the overt threats of cold war nuclear holocaust have seemingly dissipated, the underlying devices of mass destruction still exist, particularly in a world where terrorists are further spurred on by another form of technology, the mass media and internet. Such threats are unimaginable, even for those who must anticipate how to deal with the aftermath $[2,4]$.

As inferred previously, we are no longer a self-sufficient agrarian culture. Major populations are now fully dependent on day-to-day energy and water supplies, sewage, garbage removal, and food products from others. Therefore, in addition, to the initial impact of injury and illness are the subsequent public health sequelae such as insufficient food supplies, contaminated water, lack of shelter and the subsequent threat of associated diseases. Likewise, the psychological impact of disasters on populations is only just now beginning to be better appreciated, not just for those directly affected, but for the population as a whole [2]. For example, the economic downturns in the United States, Europe and elsewhere after the events of fall 2001 are often considered one of the casualties of the terrorist attacks. It emphasizes the under-recognized widespread affects that disasters can have on the international public psyche.

Even when loss of life and infrastructure are relatively minimal in the grand scheme of the particular nation involved, disasters can have significant and farreaching psychological impact (e.g., the 2001 USA anthrax postal system attacks). The mass media, internet and other modern technology not only race information to all of us worldwide, but often do so in a graphic and emotionally-stimulating manner. Even though the death and long-term morbidity toll was not as high as the staggering consequences of the Indian Ocean tsunamis, the public health risk management issues associated with the 2001 USA anthrax attacks and other identified potential threats were still enormous [2, 5, 6]. Facilitated by obsessive mass media mania, the potential threat of other public health crises (involving large populations) as a result of either terrorism or natural disease (e.g., small pox and avian flu pandemic scenarios) became more of a reality in terms of public perception. As a result, tremendous political pressure has developed for clinicians and public health officers alike to become better prepared to protect the public from all disasters, let alone doomsday scenarios.

Such psychological affects on the public play out in political venues as evidenced in the USA with the development of the Department of Homeland Security and improved worldwide intelligence-gathering cooperation both domestically and internationally. Nevertheless, while some improvements in surveillance and pharmacological resource allocations have evolved, the medical aspects of homeland security and 
public health emergency preparedness remain worrisome and clearly fall short of public expectations, appropriate expectations or not.

Sociologically, it is logical that wealthier countries might stand a better chance of mitigating mortality and morbidity with their robust health care systems, solid public infrastructures, substantial community resources and early detection-warning systems. This would occur not only through forewarned prevention of injury and illness, but also through rapid access to sustenance, medical and rescue assets. It could be emphasized that much of the loss of life and morbidity from the 2004 Indian Ocean tsunamis and their subsequent sequelae actually resulted from relative limitations in terms of such characteristics. Nevertheless, the events of the fall of 2001 in the USA, the subsequent Toronto experience with severe acute respiratory syndrome (SARS) and the recent aftermath of Hurricane Katrina in the Gulf Coast, also exposed the vulnerability of even relatively healthy and well-resourced nations.

In fact, these recent events emphasized our vulnerability in surge capacity. Regardless of disaster events, on a daily basis, the great majority of healthcare facilities and emergency medical services (EMS) systems, even in prosperous Western nations, are overwhelmed in terms of emergency care capacity, be they government-based or private entities. Many of the world's key trauma centers and EMS crews are deluged on a day-to-day basis, with in-patient facilities brimming with fully-occupied beds, sub-optimal nurse and ancillary personnel staffing, despite increasing demands for service and a higher acuity of illness and injury.

One could argue that these existing health care services, including ambulances, emergency centers, operating rooms, and intensive care units (ICUs) are facing a disaster each day as available medical resources outstrip the daily demands for urgent and critical care. This tenuous situation causes the looming threat of additional surges from disasters, be they technological or natural, to become truly deep concerns for the future of disaster medicine.

Table 1. Some key reasons for increases in the frequency, magnitude and impact of disasters (natural, unintentional and intentional) since the mid- $20^{\text {th }}$ century

Exponential growth of human populations, concentrated in high-risk venues (e.g., shorelines, earthquake zones and prior uninhabited regions), particularly those externally dependent upon on others for sustenance (food supply chains), power resources and public health hygiene, making human populations increasingly vulnerable when infrastructures disrupted.

IIncreasing exposures to zoonoses; more mutations of microbes (more human vectors); potential effects of global warming on storm formation; more sites (e.g., large buildings, subways) that are larger destructive traps.

I Technology that facilitate disasters (e.g., jet aircraft allowing more rapid spread of disease globally or terrorist use as a massive explosive device).

I Growth of hazardous industries and transportational systems (e.g., chemicals in plants, trucks, boats); genetic engineering/technology that facilitates bio-terrorism and weapons of mass effect across populations.

I Mass media (television, internet) that provide instantaneous imagery and disaster threat concerns, affecting populations psychologically worldwide, and, in turn, affecting markets/economies (also a stimulus for terrorists).

- The global, multinational, multi-jurisdictional nature of disasters because of international-global industries, businesses, and tourist activities, and the associated global vulnerability through computer/internet disruption 
In essence, there is a spiraling risk for catastrophic events involving multiple casualties and population-based medical morbidity, including proximal injury and illness and subsequent psychological and public health concerns (Table 1). Such events will likely be multinational in nature with global ramifications, even when localized to a particular venue. Therefore, this will require international cooperation in terms of prevention, mitigation and relief. However, the medical care infrastructure, even in wealthy countries already seems to operate at capacity, making a major multiple injury event or even an influenza pandemic a true challenge.

\section{Key Problems in Disaster Medicine Today}

Just as the threats of disasters have increased and become multi-faceted, so have the medical sequelae and complications $[2,4,6]$. Explosions carry the triple threat of thermal, penetrating and blunt trauma $[2,4]$. Associated building collapses not only cause crush injury syndromes, but a higher risk of being trapped, and modern fires induce chemical inhalation injuries as well as carbon monoxide poisoning and traditional respiratory tract impairment $[2,4]$. Earthquakes cause all of the above, but killer tsunamis as well because of concentrated population centers. Hurricanes, floods and tidal waves result in drowning, snake bites, and contaminated water supplies and subsequent mosquito-borne/zoonotic illnesses [2]. Chemical releases can result in pulmonary injury, burns, nerve system dysfunction, liver damage and cellular dissolution $[2,4]$. Severe radiation exposure causes burns, immunological suppression, and diffuse epithelial damage, internally and externally. Biological agents result in a myriad of physiological insults. From pneumonia, coagulopathies, and central nervous system compromise to cardiac suppression and liver failure, the viral and bacterial agents provide often-insidious challenges to clinicians and public health officers alike, regardless whether the root source is a natural epidemic or a malicious dissemination.

While using existing medical personnel would be best, developing coordinated plans to incorporate them into a disaster plan (and provide them with timely respite and staggered shifts) is the challenge. While additional personnel theoretically could be imported from nearby (unaffected) medical facilities or from other regions or countries, the local personnel work best in their own environments and still would need to provide coordinating leadership roles. This even plays out politically as was seen in the aftermath of Hurricane Katrina where local medical personnel still wanted control despite the loss of resources (hospitals, supplies), both out of sense of loyalty to 'their' patients, and their own sense of duty.

In some respects, using the local personnel is in keeping with the number one rule of multiple casualty incident management, namely to follow day-to-day routines as closely as possible or modify day-to-day routines as much as possible to meet the unique demands of a disaster [7-9]. The logic here is that unfamiliar activities or settings result in logistical and procedural learning curves for clinicians and that such medical care obstacles can be amplified in a strange venue with overwhelming patient care demands. Learning how the laboratory or pharmacy works or how to operate less familiar equipment or communications systems, can delay and impair the true focus of patient care.

In the Hurricane Katrina scenario or the Pakistani earthquake circumstance, however, much of that infrastructure was wiped out, diluting such arguments. If, then, outside teams are to be brought in, one solution is to bring in a team that al- 
ready works together well. In the aforementioned Dallas field hospital built to handle the surge from Katrina evacuees, the medical team was used to working together. Likewise, the arrival into New Orleans of the USS Comfort, a floating naval hospital replete with extensive operative capacity, neurosurgeons, multiple ICUs and airlift capacity, worked well because the team had been in place and worked well together. The question remains, however, how long could such 'borrowed teams' last in the disaster zone. What is an appropriate tour of service?

Therefore, if they are still standing and operational, the utilization of highly-experienced trauma centers and ICUs would optimize the medical care skills needed and the efficiency of the delivery of care assuming that they could be off-loaded from some of their day-to-day activities. For example, a busy emergency department could off-load sore throats, urinary tract infections, and broken arms to clinics and field infirmaries (places where surge and delays in care would have less concerning consequences). Through prospective, government-moderated agreements, ICUs at major receiving centers for disaster victims could transfer certain critical care patients to other community hospitals if necessary. This kind of arrangement has been worked out to significant degree in venues like Miami, USA, a frequent target for major hurricanes in which certain hospitals themselves are at risk of damage. In any case, there will be a need for external surge capacities outside the day-to-day facilities.

Contrary to popular perception, most disastrous events do not create an immediate influx of critical patients [7-9]. Victims are either killed outright or they have generally survivable injuries. In most traditional situations, experience has shown that less than $10 \%$ of patients (those not killed outright) will require critical care or critical care monitoring [7-10]. The main threat is actually displaced persons who need their day-to-day medical care, prescription, optometrist, dentist, and, in some cases, their dialysis or chemotherapy. In the aftermath of Hurricane Katrina, hundreds of thousands needed to be evacuated for extended periods of time. Even if most were healthy, some of these healthy evacuees were bound to have their unanticipated appendicitis or their typical sore throats. More to the point, tens of thousands, especially the elderly nursing home patients, needed so much more. And how does one address the situation of a woman who says she is due for her chemotherapy when she has no medical records or physician contact? This becomes the real challenge, not necessarily the threat from the actual disaster event. Most conventional disasters can be handled by existing resources when those resources are not involved or currently overwhelmed [8].

If the major problem becomes the destruction of the local facilities and these concerns become further confounded by legal issues such as licensure and credentialing of medical professionals coming to volunteer at other alternative hospitals or the disaster zone itself. A physician coming to provide aid at another locale or hospital may not be authorized to do so if they are not licensed to practice medicine in that jurisdiction (i.e., country, state, province). Moreover, even if licensed, hospital accreditation, in most venues, requires prospective scrutiny of physicians with relevant background checks and certain administrative requirements. All of these procedures take time and are, therefore, essentially impossible to provide at the time of a catastrophic event. While some communities have set up mechanisms to cross-credential physicians for a disaster, only a few have done so and this does not account for the issues of familiarity and skills utilization.

Furthermore, the key practitioners that might be needed to provide assistance at alternative locations in a disaster are skilled nurses, respiratory therapists, dialysis 
technicians, pharmacists and the like. While all of these practitioners could also be 'cross-credentialed', it still does not account for motivators to have any of those persons participate. Motivators are not just the dedication of a zealous volunteer or the lure of a financial incentive, but they also include care for those practitioners' families in the midst of a disaster during which time the families may be vulnerable as well (no food or water, loss of electricity, trapped by flooding, possible exposure to contagious disease, etc.). Many of the persons trapped in the flooded New Orleans hospitals were actually family members of the health care workers. Finally, there could be concerns about liability coverage and protection from malpractice lawsuits when providing services outside of one's routine location [5].

All of these concerns strengthen the argument that existing facilities, particularly major trauma centers and critical care hospitals, should all be fortified and better prepared for surge capacity. Not only are there issues of skills, experience, familiarity, learning curves, licensure, credentialing, motivation and liability protection with which to be concerned, but, again, this paradigm follows the basic disaster tenet to follow one's day-to-day routines as closely as possible [7-9]. It relies on the premise that one should prepare for such events by modifying day-to-day activities to accommodate requirements for a multi-casualty event. One of the major reasons why disaster scenarios, be they drills or actual events, often go awry is that they are encumbered by plans or procedures that fall outside normal routines. Therefore, working in environments that facilitate familiar clinical and procedural behaviors in a disaster is the most advisable strategy.

\section{Future Concerns in Disaster Medicine}

While local, reinforced facilities might be best suited for the more traditional $21^{\text {st }}$ Century disasters, a major nuclear or biological event can pose a more regional-national threat, if not a global risk [4-6]. For example, even if only a fifth of a population becomes infected with a highly contagious disease over a several week period and only $5 \%$ of such patients require critical care, this can mean 10,000 critical care patients in a city of a million residents. Assuming a week's stay for each patient whether they live or die, it still translates into the need for thousands of ICU beds at any given time. Also, under such circumstances, patients more than likely would not be transferred out to other communities because those other venues also may be experiencing similar, or even worse, surges in patient demands in such scenarios. Similarly, certain catastrophes may involve those 'routine facilities' and transport services themselves such as that seen in the Hurricane Katrina aftermath. They may be destroyed or inaccessible due to flood, earthquake, terrorist bombing or contamination. This scenario also entreats a plan for working outside one's normal routine or enormous surge at other facilities, either local or at a distance, established or makeshift.

Nevertheless, if current, day-to-day facilities are to be fortified (as they should), then these modifications will have significant fiscal impact. Already strapped with budgetary challenges, such infrastructure changes would be unreasonable without full public support (government assistance) and commitment that all other facilities would share in similar burdens. In fact, far beyond the costs of constructing better ventilation mechanisms, improved water supply systems, well-placed generators and circumferential barricades (e.g., mitigating the effectiveness of car-bombs) as well as better control of hospital entrances, are the sociological conflicts. Such security 
measures defeat the purpose and current philosophy of hospitals, clinics and medical facilities which should strive to provide even easier, patient-friendly access to medical care, particularly for the elderly, sick and chronically ill who need comfortable entrance.

Even with infrastructure changes, they will be of little avail without standardized training of the healthcare providers. During the last quarter of the $20^{\text {th }}$ Century, standardized, multi-disciplinary courses had been developed for the management of other major threats to life, namely cardiac arrest and trauma (e.g., the American Heart Association's Advanced Cardiac Life Support and the American College of Surgeons' Advanced Trauma Life Support). Such standardized training has even been demonstrated to be effective in increasing life-saving. However, unlike cardiac arrest and trauma cases that can present in certain facilities on a daily basis, disaster events are uncommon and infrequent events, even worldwide, making additional training and practice even more critical.

Even if the infrastructure is addressed and personnel trained, the complexity of nuclear explosion or 'simply' a pandemic may be overwhelming. It is feared that with the typical current processes for producing vaccines, an entirely new influenza virus could sweep through even healthy worldwide populations in a matter of months, long before a vaccine could be developed, processed, and distributed, not to mention the time it takes for inoculated persons, particularly children, to develop adequate protective antibodies. A similar genetic jump from animal populations (e.g., 'avian flu') could proliferate with the same scenarios. In both cases, with more people to infect, more ways of rapidly transmitting the virus around the globe, and more persons with immunological suppression alive today, the risk for pandemic will become even more of a threat, not only for the population as a whole, but also for the ambulance crews, emergency department staff and ICU practitioners. If they become ill as well, there will be even fewer healthcare providers available to care for the throngs of ill persons, making the disaster scenario even worse. Such as seen in the SARS situation, the medical facilities and healthcare workers may become a main vector themselves.

More worrisome is the unknown. The concept of SARS was unknown three years ago. With tremendous advances in genetic engineering, it is feared that both malicious and unintentional contagion threats will become new realities. And while threats for disaster are increasing, medical care resources are being spread thinner and thinner, from sociological factors (nursing shortages) to financial constraints (decreasing reimbursements for medical care).

Ironically, with increased demands for protection from terrorism and other public health threats, financial resources have been diverted from healthcare to national defense and homeland security efforts. Unfortunately, most security efforts are overhead costs and not at all revenue-generating. Even within hospitals, dealing with disaster management is generally administrative in nature (training, equipment, procedures, personnel) and consumes and diverts medical care professionals' time and efforts from their day-to-day patient care activities. These concerns are likely to become worse and resources further drained. Moreover, the actual costs of the disasters are accelerating and public expectation for assistance stronger than ever before. 


\section{Some Potential Solutions and Improved Preparations}

Many lessons have been learned in our era of expanding and more frequent disasters. In a cyclone, tornado or hurricane belt, reinforced hospitals should have their ICUs and operating rooms secured within the center of the edifice, well above the level of potential storm surges and flood levels and far below the roof-top levels where spin-off tornadoes can rip off the top floors and blow out exterior windows. Secondary and back-up generators should be protected in a similar fashion. All hospitals, particularly those in recognized earthquake zones, should have 'earthquake-proof' designs with applicable structural integrity. When a hospital is a potential terrorist target such as any main receiving facility, trauma center, burn center, or children's hospital, security measures will dictate the need for barricades to bombs and specialized ventilation systems that detect and can control the spread of aerosolized poisons or biologicals. These hospitals should have a disproportionate number of negative pressure rooms and well-established decontamination zones around the potential entrance and receiving personnel (e.g., trained triage nurses and security personnel will be staged further out into the periphery and prepared with universal precautions and easy access to decon (decontamination) suits, including high level personal protective equipment (PPE). Within the next ten years, spurred on by additional major terrorist events and further recognition of the vulnerability of the medical safety net, governmental resources will likely begin to make these changes and architects for new facilities will incorporate them into future design. But, economically intensive, this aspect of preparation will lag behind other efforts.

This first consideration to enhance the structural aspects of disaster management would be of most value to handle the more likely conventional disasters. However, one might then return to entertaining the concept of a specialized facility or facilities to manage pandemics and disseminated bioterrorism incidents such as a mutated smallpox organism. In this case, to overcome the concepts of lack of familiarity with equipment and resources, credentialing, liability and medical skills utilization, a mitigating solution would be to dedicate a reserve team of medical personnel in the way a government entity deploys a fire service or army as a dedicated standing force. Currently, a hybrid for this type of concept is accomplished through the mobile medical teams and tent hospitals designed by the National Disaster Medical System (NDMS), an element of the Federal Emergency Management Agency (FEMA) in the United States Department of Homeland Security. Although this does not employ a round-the-clock standing team, it does utilize a 'stand-by' team of medical and allied health volunteers who are trained and routinely exercised to operate mobile hospitals. Currently, these teams would likely be inadequate for a mass casualty scenario with tens of thousands of victims, but still they are somewhat helpful in areas where the standing facilities have been destroyed, impaired or are inaccessible. The Hurricane Katrina incident demonstrated the clear value of expert, co-trained military medicine assets.

Mobile hospitals have reasonable value in some circumstances and should always be part of the planning including safe and secured storage of required equipment and assets, but community-wide plans to enhance existing facilities for surge capacity should still supercede all other plans. Inter-hospital agreements to facilitate transfers of patients to balance out surges throughout the system should be in play in the best prepared communities. Such agreements will also include specialized storage facilities for antidotes and antibiotics, perhaps in a separate, undisclosed 
and protected storage facility, but close enough for easy access. The regional hospitals should band together and purchase these items in bulk to leverage economies of scale (cheaper prices), but they should also coordinate receipt so that expiration dates on the drugs will be staggered and not all expiring at once. Such coordination would also include the use of healthcare and ancillary personnel in case of overload at a given hospital or incapacity of another.

Not only will prospective cross-credentialing be accomplished ahead of time using familiar mechanisms, but the process may even be facilitated by course completion in Advanced Disaster Life Support and Hospital Disaster Life Support types of courses $[1,2,11]$. In all likelihood, arrangements should first focus intra-murally. For example, they may prioritize the use of nurses from the same hospital system (i.e., many hospital systems operate more than one hospital) because of the similarities in policies, procedures, liability coverage and payroll considerations. Such coordination of efforts would be a massive under-taking, but well-prepared communities will want to cooperate in such approaches. Also, since many disasters are multinational and often occur in under-resourced countries with relatively poor infrastructures, the creation of dozens of international disaster teams by the United Nations or other organizations worldwide could be considered. Equipped with fast transport aircraft and mobile emergency units, these teams would best be available on any site within less than 6 hours and will work closely with local organizations. Very likely, each disaster team will be staffed and operated by a multinational force, and most likely a standing military team. As in any other military organization, teaching and training in disaster medicine will be part of military education in all countries and the teams will continuously drill and work together in coordination on a routine basis. If anything has been learned in recent disasters, it has been the clear value of the military.

Another lesson learned was the value of standardized training, not only for the military, but for the civilian population as well. After 2001, the American Medical Association (AMA), working with several major academic centers, began to help development of a family of standardized, interoperable, multi-disciplinary, all-hazards courses to deal with the medical aspects of disaster medicine and counter-terrorism [1-3]. Working in close conjunction with university-based trauma center teams as well as multiple federal and military agencies and, more recently, other professional societies such as the American College of Emergency Physicians (ACEP) and the Society of Critical Care Medicine (SCCM), the AMA courses are beginning to lay the groundwork for standardized training and improved personnel preparations for disasters $[1-3,11]$. During Hurricane Katrina, the Louisiana State Department of Health attributed many of the medical evacuations that were accomplished quite successfully, to the use (and previous training in) ADLS as well the use of a table top disaster scenario called "Hurricane Pam". Like Advanced Cardiac Life Support and Advanced Trauma Life Support, the Advanced Disaster Life Support course provides hands-on, multi-disciplinary scenarios in which participants learn to provide antidotes and other resuscitative skills in simulated austere, hazardous conditions requiring the donning and use of high-level PPE in insecure environments [1]. In addition to the Advanced Disaster Life Support course, a prerequisite Basic Disaster Life Support course [2] provides intensive exposure to the didactic elements of disaster preparation, be it chemical, biological, radiological, or traumatic in nature $[2,4]$. Within the next ten years, it is predicted that the Basic Disaster Life Support course will be required for every medical student, paramedic student, nursing and other applicable allied health personnel in the USA [2]. The 
Advanced Disaster Life Support course will also be provided to applicable trainees and nursing staff in critical care areas including advanced life support ambulances, emergency departments, and critical care areas. It is also predicted that specialized in-hospital spin-off courses (e.g., Hospital Disaster Life Support) will address elements of decontamination tactics by custodians, engineers and others. The SCCM has already laid much of the groundwork for such training with some of their course developments [11]. Such endeavors will hopefully become similar to the efforts conducted by the International Liaison Committee on Resuscitation (ILCOR) for cardiac resuscitation medicine using evidence-based approaches and worldwide consensus.

In addition to being involved in such standardized training, paramedics and emergency medical technicians should also be trained to deliver prophylaxis in a public emergency preparedness situation such as a smallpox or yersinia outbreak or even an influenza pandemic. Prospective rules about who can be denied or provided vaccination or antibiotics and how one receives protection from liability should be addressed ahead of time in the most prepared systems. In addition to healthcare workers, certain societal infrastructure personnel (e.g., elected officials, water, power, communications, media personnel) may be prioritized as well. Also, anticipating a major event, facilities should develop a cadre of antidotes, antivirals and antibiotics for biological threats. More importantly, they will need to ratchet up ICU equipment, ventilators and respiratory care equipment as well as PPE and decontamination equipment. Ambulances and emergency departments and hospitals at large should be fortified by new computerized technology that enhances detection and discrimination of abnormal gases, chemicals, aerosolized biologicals and other threats in ventilation systems and ambient air, just as a carbon monoxide detector or Geiger counter would provide sentinel detection of carbon monoxide or radioactivity in one's home. Medical records will be electronically stored and also duplicated and stored at distant sites in case of medical facility destruction and population displacements. New ventilators will need to be impervious to chemical and biological agents and provide protected ventilation in such environments. In addition, artificial hemoglobin-based oxygen carriers that can be stored in ambulances or in far-forward military conditions should be researched and eventually placed in massive storage places as well and ready to use for mass casualties [12]. Some products now in early test stages can be stored without refrigeration for several years and are likely to be standard equipment in all critical care settings, be it prehospital, emergency center or ICU settings [12].

Again, all of this will require a new level of international cooperation particularly because many natural disasters can be superimposed upon on-going complex emergencies, including on-going famines, civil wars or rebel insurgencies [13]. Such circumstances can further complicate rescue and restoration of normalcy [13].

\section{Conclusion}

There is a worldwide spiraling risk for more frequent catastrophic events involving multiple casualties, not only in terms of acute injury and illness, but also subsequent psychological and public health concerns. Today, such events will likely be multinational in nature, even when localized to a particular venue and this require international cooperation in terms of prevention, mitigation and relief. The best approach to preparing for disasters is to expand, modify and enhance current local 
infrastructures and capabilities for managing the multiple types of disaster scenarios and create a number of inter-facility cooperative agreements in advance. Aside from safer internal locations for ICUs and surgical theaters, certain structural changes will need to be installed such as modified ventilation systems, protected water supplies, decontamination mechanisms and security renovations. A key strategy will be to proliferate interoperable, multi-disciplinary, all-hazards training initiatives such as the AMA National Disaster Life Support courses. Purchases of cadres of antidotes, antibiotics and hemoglobin-based oxygen carriers should be coordinated regionally, stored in secure locations and made readily-available for the applicable disaster scenario.

\section{References}

1. American Medical Association (2003) Advanced Disaster Life Support ${ }^{\circledR}$, Version 2.0. American Medical Association Press, Chicago

2. American Medical Association (2002) Basic Disaster Life Support ${ }^{\circledR}$, Version 2.0. American Medical Association Press, Chicago

3. American Medical Association (2004) Core Disaster Life Support ${ }^{\circledR}$, Version 2.0. American Medical Association Press, Chicago

4. Keyes DC, Burstein JL, Schwartz RB, Swienton RE (2004) Medical Response to Terrorism: Preparedness and Clinical Practice. Lippincott, Williams and Wilkins, Philadelphia

5. Pepe PE, Rinnert KJ (2002) Bioterrorism and medical risk management. The International Lawyer 36:9-20

6. Bartlett JG (2002) Bioterrorism and Public Health, $1^{\text {st }}$ Edition. Thomson Medical Economics, Montvale

7. Pepe PE, Stewart RD, Copass MK (1989) Ten golden rules for urban multiple casualty incident management. Prehosp Disaster Med 4:131-134

8. Pepe PE, Anderson E (2001) Multiple casualty incident plans: ten golden rules for prehospital management. Dallas Med Journal, November: 462-468

9. Pepe PE (1990) Responding to emergencies - review of the Phillips Petroleum explosion, March issue. Council of State Governments, Louisville, p 9

10. Pepe PE, Kvetan V (1991) Field management and critical care in mass disaster. Crit Care Clin 7:401-420

11. Farmer JC, Jiminez EJ, Rubinson L, Talmor DS, Markenson DS (2003) Fundamentals of Disaster Management, $2^{\text {nd }}$ Edition. Society for Critical Care Medicine, Des Plaines

12. Manning JE, Katz LM, Brownstein MR, et al (2000) Bovine hemoglobin-based oxygen carrier (HBOC-201) for resuscitation of uncontrolled, exsanguinating liver injury in swine. Shock 13:152-159

13. Spiegel PH (2005) Differences in world responses to natural disasters and complex emergencies. JAMA 293:1915-1918 